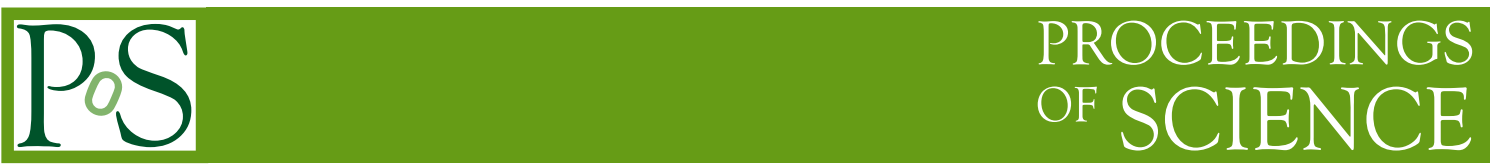

\title{
New physics searches through $\tau$ decays at Belle
}

\author{
Sourav Patra \\ (on behalf of Belle Collaboration) \\ Indian Institute of Science Education and Research Mohali, \\ Punjab, India
}

E-mail: souravpatra3012@gmail.com

We report the result of a search for $\tau^{ \pm} \rightarrow \ell^{ \pm} \gamma(\ell=\mu, e)$ using the full data sample at Belle. Since the observation of neutrino oscillations has unambiguously shown that the lepton flavor is no longer conserved, we can expect lepton flavor violation (LFV) in the charged lepton sector. Though the standard model (SM) does not predict charged LFV decays at an observable rate, $\tau^{ \pm} \rightarrow \ell^{ \pm} \gamma$ is predicted by many new physics scenarios and is thus one of the most promising LFV modes. Consequently, we have obtained the most stringent limit on the branching fraction of $\tau^{ \pm} \rightarrow \mu^{ \pm} \gamma$. In addition, we report the result of a search for tau electric dipole moment (EDM) evaluating $u$ vertex coupling using the full data sample at Belle. At present, the observed CP violation is insufficient to explain the prevalent matter-antimatter asymmetry in our universe. On the other hand, the EDM of leptons is predicted to be negligibly small in the SM and is expected as a source of CPV in the lepton sector induced by some new physics. We have obtained one order more sensitive result both for the real and imaginary parts of the $\tau$ EDM.

\footnotetext{
*** The European Physical Society Conference on High Energy Physics (EPS-HEP2021), *** ***26-30 July $2021 * * *$

*** Online conference, jointly organized by Universität Hamburg and the research center DESY ***
} 


\section{Introduction}

Tau is the heaviest lepton in the standard model (SM). Plenty of new physics phenomenons like lepton flavor violation, lepton number violation, baryon number violation, $\mathrm{CP}$ violation are possible to study in various tau decays. LFV decays are forbidden in the SM but occurs with a yet unobservably small probability, $O\left(10^{-40}\right)$, via neutrino oscillations [1]. However, several theories beyond standard model (BSM) such as SUSY, GUT, seesaw mechanisms predict enhancement of the LFV transitions in $\tau^{ \pm} \rightarrow \ell^{ \pm} \gamma(\ell=\mu, e)$ decays to the current experimental sensitivity [2-4]. An observation of LFV would be a clear signature of BSM physics. Also, the electric dipole moment of the $\tau$ lepton is a physical parameter that parameterizes CP violation at the $\gamma \tau \tau$ vertex. In the SM, existence of $\tau$-lepton EDM is unobservably small ( $\left.\sim 10^{-37} \mathrm{ecm}\right)$ [5]. However, some new physics models indicate a larger EDM of order $10^{-19} \mathrm{ecm}[6]$. Hence, observation of a non-zero $\tau$ EDM is a clear sign of new physics phenomena. Belle detector [7] was installed at the KEKB asymmetric $e^{+} e^{-}$collider [8].

\section{Search for LFV in $\tau^{ \pm} \rightarrow \ell^{ \pm} \gamma$ decays}

We update the search for LFV in $\tau^{ \pm} \rightarrow \ell^{ \pm} \gamma$ decays [9] using $988 \mathrm{fb}^{-1}$ of Belle data corresponding to 912 million of $\tau$-pair events. Signal Monte Carlo (MC) samples and generic $\tau^{+} \tau^{-}$processes are generated by KKMC and TAUOLA [10]. A $\tau^{+} \tau^{-}$pair event is divided into two hemispheres, signal-side and tag-side tau, in the CM frame using a thrust vector [11]. The signal-side tau decays to a muon (electron) and a photon for the $\tau^{ \pm} \rightarrow \mu^{ \pm} \gamma\left(\tau^{ \pm} \rightarrow e^{ \pm} \gamma\right.$ ) search. The number of photons in the signal side should be exactly one. The tag-side tau is assumed to undergo one-prong decays such as $\tau \rightarrow e v \bar{v}, \mu \nu \bar{v}, \pi v$ and $\rho v$ and named as $e, \mu, \pi$ and $\rho$ channel, respectively.

A missing-mass-squared on the tag side $\left(m_{v}^{2}\right)$ is defined as $m_{v}^{2}=\left(E_{\ell \gamma}^{\mathrm{CM}}-E_{\mathrm{tag}}^{\mathrm{CM}}\right)^{2}-\left|\vec{p}_{\mathrm{miss}}^{\mathrm{CM}}\right|^{2}$ where, $E_{\ell \gamma}^{\mathrm{CM}}\left(E_{\mathrm{tag}}^{\mathrm{CM}}\right)$ is the sum of the energy of the signal (tag) side in the CM frame and $\left|\vec{p}_{\text {miss }}^{\mathrm{CM}}\right|$ is the magnitude of the missing momentum in CM frame. The shape of the $m_{v}^{2}$ distribution depends on the number of the neutrinos on the tag side. The $m_{v}^{2}$ requirements for $e / \mu, \pi$ and $\rho$ channels within $[0.0,2.8],[-0.1,1.2]$ and $[-0.3,1.5] \mathrm{GeV}^{2}$ (assuming $c=1$ here and all other places) respectively are applied to reduce the $\tau^{+} \tau^{-}$background. To improve the sensitivity, two more variables are introduced. The first one is an energy asymmetry between the lepton and the photon in the signal side, $\left|E_{\ell}^{\mathrm{CM}}-E_{\gamma}^{\mathrm{CM}}\right| /\left(E_{\ell}^{\mathrm{CM}}+E_{\gamma}^{\mathrm{CM}}\right)$, requiring the energy asymmetry less than 0.65 . The second variable $\xi_{\tau(\text { tag),track(tag) }}^{\mathrm{CM}}$ is defined as:

$$
\xi_{\tau(\text { tag }) \text { track (tag) }}^{\mathrm{CM}}=\frac{\vec{p}_{\tau(\mathrm{tag})}^{\mathrm{CM}} \vec{p}_{\text {track(tag) }}^{\mathrm{CM}}}{\left|\vec{p}_{\tau(\text { tag })}^{\mathrm{CM}}\right|\left|\vec{p}_{\text {track(tag) }}^{\mathrm{CM}}\right|}
$$

where, $\vec{p}_{\tau(\text { tag })}^{\mathrm{CM}} \cdot \vec{p}_{\text {track (tag) }}^{\mathrm{CM}}=\left(m_{\text {miss }}^{2}-m_{\tau(\mathrm{tag})}^{2}-m_{\text {track }}^{2}+\sqrt{s} E_{\text {track }(\mathrm{tag})}\right) / 2$ with $m_{\text {miss }}^{2}$ as missing mass squared of the event against the lepton and the photon in signal side and tag-side track and $\sqrt{s}$ as the total energy in the CM frame. Distributions of $\xi_{\tau(\operatorname{tag}) \text { track(tag) }}^{\mathrm{CM}}$ are shown in the Fig. 1. Huge $\tau \tau$ and other backgrounds are removed by selecting $0<\xi_{\tau(\text { tag),track(tag) }}^{\mathrm{CM}}<1$. The signal region is defined by two kinematic variables: i) beam-constrained-mass, $M_{\mathrm{bc}}=\sqrt{\left(E_{\mathrm{beam}}^{\mathrm{CM}}\right)^{2}-\left|\vec{p}_{\ell \gamma}^{\mathrm{CM}}\right|^{2}}$ and ii) the normalized energy difference, $\Delta E / \sqrt{s}$ where $\vec{p}_{\ell \gamma}^{\mathrm{CM}}$ is a sum of the lepton and photon momenta in 

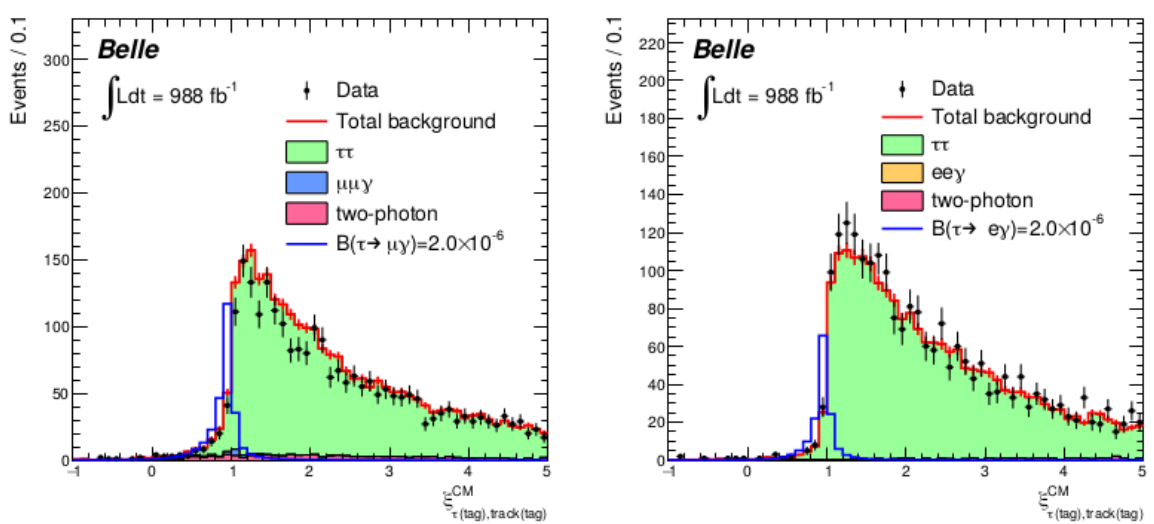

Figure 1: Distribution of $\xi_{\tau(\text { tag),track(tag) }}^{\mathrm{CM}}$ for $\tau^{ \pm} \rightarrow \mu^{ \pm} \gamma$ (left) and $\tau^{ \pm} \rightarrow e^{ \pm} \gamma$ (right) channels. The blue histograms show the signal MC samples with an assumed branching fraction $\mathcal{B}\left(\tau^{ \pm} \rightarrow \ell^{ \pm} \gamma\right)=2.0 \times 10^{-6}$ [9].

the CM frame. The elliptical signal regions are selected with $\left[\left(M_{\mathrm{bc}}-\mu_{M_{\mathrm{bc}}}\right) / 2 \sigma_{M_{\mathrm{bc}}}\right]^{2}+[(\Delta E / \sqrt{s}-$
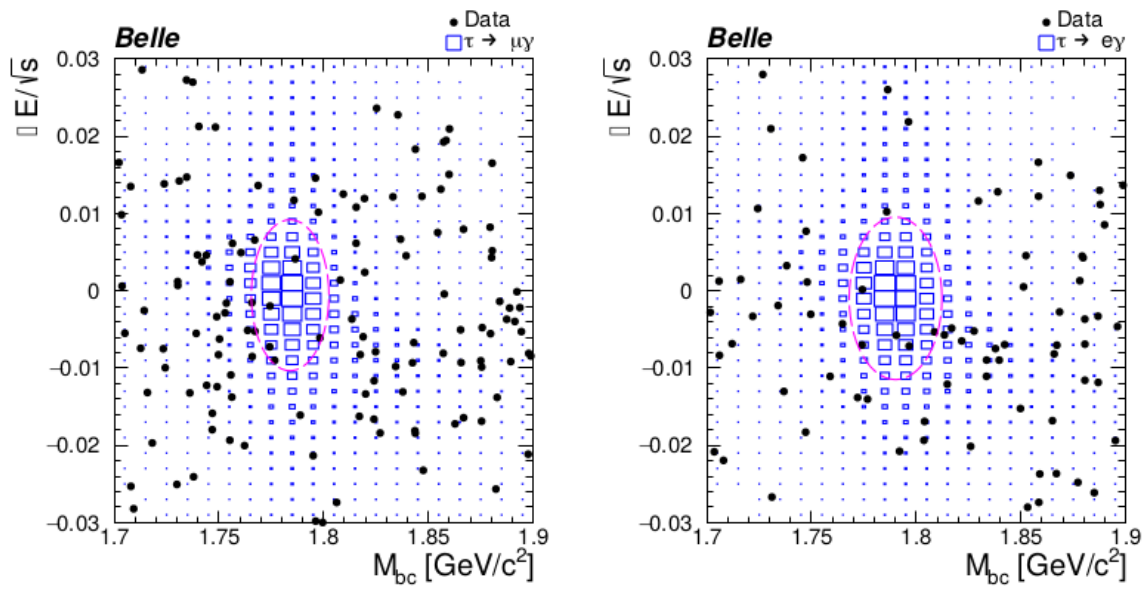

Figure 2: $\Delta E / \sqrt{s}$ vs $M_{\mathrm{bc}}$ distribution for $\tau^{ \pm} \rightarrow \mu^{ \pm} \gamma$ (left) and $\tau^{ \pm} \rightarrow e^{ \pm} \gamma$ (right) events. Black points are data, blue squares are $\tau^{ \pm} \rightarrow \ell^{ \pm} \gamma$ signal MC events, and magenta ellipses show the signal region used in this analysis ( $\pm 2 \sigma$ region) [9].

$\left.\left.\mu_{\Delta E / \sqrt{s}}\right) / 2 \sigma_{\Delta E / \sqrt{s}}\right]^{2}<1$ where $\mu$ and $\sigma$ are taken from the MC signal fits. Signal distributions are shown in the Fig. 2.

The analysis uses unbinned maximum-likelihood fit (inside the signal region) for the signal extraction. Estimated signal efficiency for $\tau^{ \pm} \rightarrow \mu^{ \pm} \gamma$ and $\tau^{ \pm} \rightarrow e^{ \pm} \gamma$ are $3.7 \%$ and $2.9 \%$ respectively. The expected yield of background events is 5.8 $\pm 0.4(5.1 \pm 0.4)$ for $\tau^{ \pm} \rightarrow \mu^{ \pm} \gamma\left(\tau^{ \pm} \rightarrow e^{ \pm} \gamma\right)$ mode. For both LFV modes, we observe 5.0 events. Systematic uncertainties from various sources like track reconstruction efficiency, photon reconstruction efficiency, photon energy calibration, integrated luminosity, trigger efficiency, background model are consider as potential sources of systematic. Adding individual the contributions from different sources in a quadrature, expected systematic uncertainty for $\tau^{ \pm} \rightarrow \mu^{ \pm} \gamma$ and $\tau^{ \pm} \rightarrow e^{ \pm} \gamma$ modes are $6.2 \%, 6.5 \%$, respectively. In absence of any significant signal, we estimate the upper limit (UL) of branching fractions in a frequentist approach 
using toy MC simulations. The estimated ULs of branching fraction at $90 \%$ confidence level (CL) are $\mathcal{B}\left(\tau^{ \pm} \rightarrow \mu^{ \pm} \gamma\right)<4.2 \times 10^{-8}$ and $\mathcal{B}\left(\tau^{ \pm} \rightarrow e^{ \pm} \gamma\right)<5.6 \times 10^{-8}$. Our upper limit for $\tau^{ \pm} \rightarrow \mu^{ \pm} \gamma$ is the most stringent limit to the date.

\section{Search for EDM of $\tau$ lepton}

The effective Lagrangian for $\tau$-pair production including the EDM term at the $\gamma \tau \tau$ vertex [12] is,

$$
\mathcal{L}=\bar{\tau}\left[-e Q \gamma^{\mu} A_{\mu}-\iota d_{\tau} \sigma^{\mu \nu} \gamma_{5} \partial_{\mu} A_{v}\right] \tau
$$

Corresponding the squared spin density matrix for the production vertex in the process $e^{+} e^{-} \rightarrow \tau^{+} \tau^{-}$ is given by,

$$
\mathcal{M}_{e^{+} e^{-} \rightarrow \tau^{+} \tau^{-}}^{2}=\mathcal{M}_{\mathrm{SM}}^{2}+\operatorname{Re}\left(d_{\tau}\right) \mathcal{M}_{\mathrm{Re}}^{2}+\operatorname{Im}\left(d_{\tau}\right) \mathcal{M}_{\mathrm{Im}}^{2}+O\left(d_{\tau}^{2}\right)
$$

where $\mathcal{M}_{\mathrm{SM}}$ is the SM term, and $\mathcal{M}_{\mathrm{Re}}, \mathcal{M}_{\mathrm{Im}}$ are the interference terms between the SM and EDM. These matrix elements can be expressed in terms of momentum of initial $e^{ \pm}$and outgoing $\tau^{ \pm}$ along with the spin of the $\tau^{ \pm}$. To improve the sensitivity of the EDM searches, optimal variables, $O_{\mathrm{Re}}=\frac{\mathcal{M}_{\mathrm{Re}}^{2}}{\mathcal{M}_{\mathrm{SM}}^{2}}$ and $O_{\mathrm{Im}}=\frac{\mathcal{M}_{\mathrm{Im}}^{2}}{\mathcal{M}_{\mathrm{SM}}^{2}}$, have been used in this analysis. The mean values of these observables $\left(\left\langle O_{\operatorname{Re}}\right\rangle,\left\langle O_{\mathrm{Im}}\right\rangle\right)$ are linearly dependent on the real and imaginary parts of $d_{\tau}$ with the following equations.

$$
\left\langle O_{\operatorname{Re}}\right\rangle=a_{\operatorname{Re}} \operatorname{Re}\left(d_{\tau}\right)+b_{\operatorname{Re}} \quad\left\langle O_{\mathrm{Im}}\right\rangle=a_{\mathrm{Im}} \operatorname{Im}\left(d_{\tau}\right)+b_{\mathrm{Im}}
$$

Coefficient $a_{\mathrm{Re} / \mathrm{Im}}$ (sensitivity) and $b_{\mathrm{Re} / \mathrm{Im}}$ (offset) are determined by using the MC samples.

We use $833 \mathrm{fb}^{-1}$ of Belle data in this analysis. Eight different final states with $\tau \tau \rightarrow(e v \bar{v})(\mu \nu \bar{v})$, $(\mu v \bar{v})(\pi v),(\mu v \bar{v})(\rho v),(e v \bar{v})(\pi v),(e v \bar{v})(\rho v),(\pi v)(\pi v),(\rho v)(\rho v)$ and $(\pi v)(\rho v)$ decays have been considered and those are named as $e \mu, \mu \pi, \mu \rho, e \pi, e \rho, \pi \pi, \rho \rho$ and $\pi \rho$ modes, respectively. $\rho^{ \pm}$has been reconstructed from a charged track and a $\pi^{0}$. Charged tracks are selected with a transverse momentum greater than $0.1 \mathrm{GeV} / c$ in the lab frame and photons with energy in the lab frame greater than $0.1 \mathrm{GeV}$. To suppress the background from two-photon event, we require the missing momentum of the event not be directed along the beam pipe, $-0.950<\cos \theta_{\text {missing }}<0.985$.

To calculate the optimal observables, one need to know the $\tau$ spin vectors and those spin vectors are depending on the $\tau$ flight directions. Due to the presence of one or more missing neutrinos, $\tau$ flight direction can not be determined experimentally. For the $\tau \tau$ final states with two neutrinos (like $\pi \pi, \rho \rho$ and $\pi \rho$ modes), the tau directions can be determined with a twofold ambiguity. Having one leptonic decay in the final state put one more ambiguity. Those ambiguities are resolved by taking the most probable (instead of actual) spin direction by averaging over the possible solutions. The relation between the EDM and the mean values of the observables, $\left\langle O_{\operatorname{Re} / \mathrm{Im}}\right\rangle$, are evaluated using MC simulation for various values of the EDM. By fitting the relation with a linear function, the coefficients $a_{\mathrm{Re} / \mathrm{Im}}$ and $b_{\mathrm{Re} / \mathrm{Im}}$ are obtained (shown in Fig. 3). Non zero values of $b_{\mathrm{Im}}$ arises due to non-uniform acceptance of the detector. As one can see from the values of $a_{\mathrm{Re} / \mathrm{Im}}$, the $\pi \rho$ and $\rho \rho$ modes have the highest sensitivities for $\tau$ EDM measurement.

Although the agreement between data and MC was good, we estimate the systematic errors from different sources like detector alignment, momentum reconstruction, detector response on the particle charge, mismatch in the data-MC distribution, variation in the background yield, effect of 

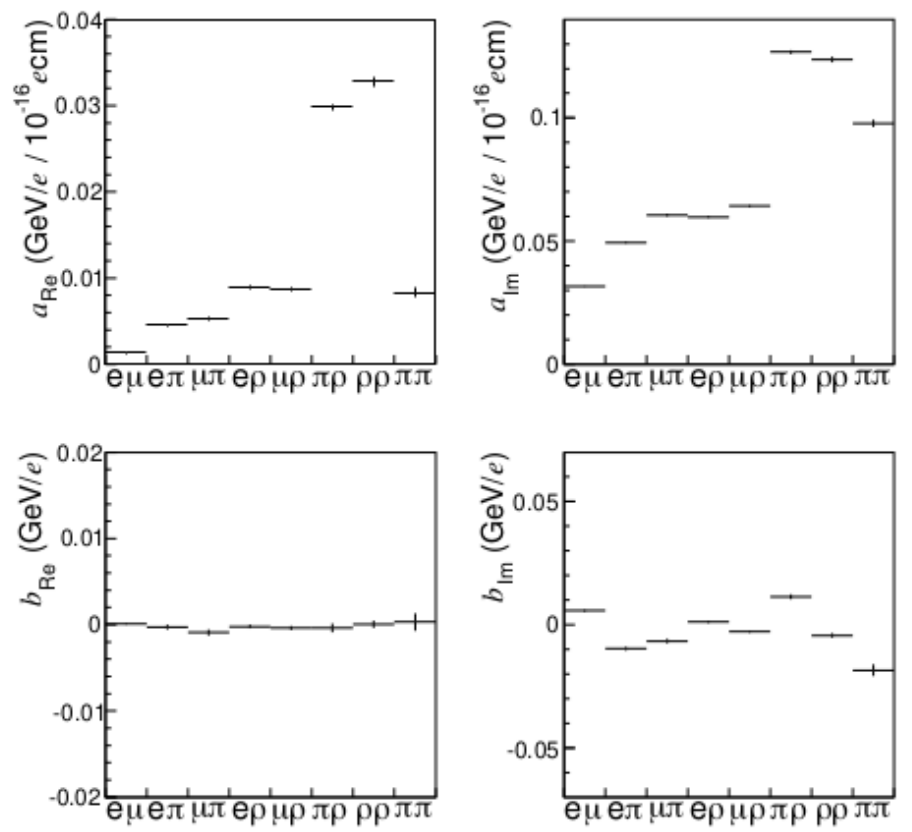

Figure 3: The EDM parameter sensitivity $a_{\mathrm{Re} / \mathrm{Im}}$ (top) and offset $b_{\mathrm{Re} / \mathrm{Im}}$ (bottom) for each mode obtained by MC. The errors are due to the MC sample size [13].

initial state radiations. Systematic for the real (imaginary) part of $d_{\tau}$ for various modes ranges from 0.9 to 5.2 ( 0.4 to 2.6 ) in the units of $10^{-17} e \mathrm{~cm}$. Finally, we obtain the mean values of $\tau$ EDM for the real and imaginary parts:

$$
\operatorname{Re}\left(d_{\tau}\right)=(-6.2 \pm 6.3) \times 10^{-18} e \mathrm{~cm} \quad \operatorname{Im}\left(d_{\tau}\right)=(-4.0 \pm 3.2) \times 10^{-18} e \mathrm{~cm}
$$

where statistical and systematic errors are summed quadratically. These results are one magnitude order more stringent as compared to the previous results [12].

\section{Summary}

We search for LFV transition in $\tau^{ \pm} \rightarrow \ell^{ \pm} \gamma$ decays. In absence of any significant signal, ULs of branching fraction at $90 \% \mathrm{CL}$ are estimated to be $\mathcal{B}\left(\tau^{ \pm} \rightarrow \mu^{ \pm} \gamma\right)<4.2 \times 10^{-8}$ and $\mathcal{B}\left(\tau^{ \pm} \rightarrow e^{ \pm} \gamma\right)<5.6 \times 10^{-8}$. Our result for $\tau^{ \pm} \rightarrow \mu^{ \pm} \gamma$ mode is most stringent to the date. Also, we report a preliminary measurement on $\tau$ EDM. New measurements of the EDM parameters are one magnitude order more stringent with respect to the current results.

\section{References}

[1] X. Y. Pham, Eur. Phys. J. C 8, 3 (1999).

[2] A. Brignole and A. Rossi, Nucl. Phys. B 701, 3 (2004). 
[3] L. Calibbi, A. Faccia, A. Masiero, and S. K. Vempati, Phys. Rev. D 74, 116002 (2006).

[4] J. R. Ellis, J. Hisano, M. Raidal, and Y. Shimizu, Phys. Rev. D 66, 115013 (2002).

[5] M. J. Booth, hep-ph/9301293; U. Mahanta, Phys. Rev. D 54, 3377 (1996).

[6] W. Bernreuther, A. Brandenburg, and P. Overmann, Phys. Lett. B 391413 (1997).

[7] A. Abashian et al., Nuclear Instruments and Methods in Physics Research A 479117232 (2002); also see Section 2 in J. Brodzicka et al., Prog. Theor. Exp. Phys. 2012, 04D001 (2012).

[8] S. Kurokawa, E. Kikutani, Nuclear Instruments and Methods in Physics Research A 499001 007 (2003), and other papers included in the volume; T. Abe et al., Prog. Theor. Exp. Phys. 2013 03A001 (2013) and following articles up to 03A011.

[9] K. Uno et al. (Belle Collaboration), J. High Energ. Phys. 2021, 19 (2021)

[10] S. Jadach, B. F. L. Ward, and Z. Was, Comput. Phys. Commun. 130, 260 (2000).

[11] S. Brandt, C. Peyrou, R. Sosnowski, and A. Wroblewski, Phys. Lett. 12, 57 (1964); E. Farhi, Phys. Rev. Lett. 39, 1587 (1977).

[12] K. Inami et al. (Belle Collaboration), Phys. Lett. B 551, 16 (2003)

[13] K. Inami et al. (Belle Collaboration), arXiv:2108.11543 [hep-ex]. 